\title{
9 Globale Gesundheit
}

\section{Matthias Egger, Nicola Low, Oliver Razum}

Durch die Globalisierung werden wir zunehmend mit Problemen konfrontiert, welche Landesgrenzen überschreiten. Auch viele gesundheitspolitische Entscheidungen werden heute auf europäischer Ebene oder unter der Mitarbeit internationaler Organisationen getroffen.

In diesem Kapitel betrachten wir Gesundheitsindikatoren sowie Krankheits- und Todesursachen im Hinblick auf das Bevölkerungseinkommen und die Entwicklung in verschiedenen Ländern. Wir analysieren die wichtigsten Faktoren, die die Gesundheit der Menschen in Industrie- und Entwicklungsländern beeinflussen und beschäftigen uns schließlich mit den Strategien und Akteuren, welche die Globale Gesundheit heute prägen.

Schweizerische Lernziele: CPH 46-48

\subsection{Internationale Vergleiche}

\subsubsection{Klassifizierung der Länder nach Einkommen und Entwicklung}

Der Forschungsbereich Globale Gesundheit beschäftigt sich vor allem mit Analysen und Vergleichen des Gesundheitsstatus' von Bevölkerungen in verschiedenen Ländern unter Berücksichtigung des jeweiligen soziokulturellen und ökonomischen Kontexts. Hierzu werden die Länder meist nach ihrem Bruttonationaleinkommen oder nach dem Entwicklungsstand klassifiziert. Häufig verwendet man dabei die von der Weltbank definierten vier Einkommensgruppen (High income, Upper middle income, Lower middle income, Low income) sowie die Kriterien der Vereinten Nationen für den Entwicklungsstand eines Landes (s. Box 9.1.1). 
Box 9.1.1: Einteilung der Länder nach Einkommen und Entwicklung [Daten von 2009].

Die Weltbank klassifiziert die Länder nach ihrem Bruttonationaleinkommen pro Kopf und Jahr (Gross National Income per capita).

- High-income-Länder:

$$
>12.195 \text { US-Dollar }
$$

- Upper-middle-income-Länder:

$$
\text { 3.946-12.195 US-Dollar }
$$

- Lower-middle-income-Länder:

$$
\text { 996-3.945 US-Dollar }
$$

- Low-income-Länder:

$$
<996 \text { US-Dollar }
$$

Bei den High-income-Ländern wird unterschieden zwischen den Industrienationen der OECD (Organisation für wirtschaftliche Zusammenarbeit und Entwicklung) und anderen High-income-Ländern, wie z. B. den Ölförderländern am Arabischen Golf.

Die Vereinten Nationen (UN) bezeichneten 2009 insgesamt 49 Länder aufgrund der folgenden Kriterien als Least Developed Countries (LDC):

- Niedriges Pro-Kopf-Einkommen: durchschnittliches jährliches Bruttonationaleinkommen über drei Jahre < 905 US-Dollar

- Hohe ökonomische Verwundbarkeit: basierend auf dem Economic Vulnerability Index, der u.a. den Anteil der verarbeitenden Industrie und der Dienstleistungen sowie die Stabilität der landwirtschaftlichen Produktion und des Exports von Gütern und Dienstleistungen erfasst

- Geringe humane Ressourcen: basierend auf dem Human Assets Index, der auf Angaben zur Alphabetisierungsrate, zur Einschulungsrate in Sekundarschulen, zur Ernährungslage und zur Kindersterblichkeit beruht

Tab. 9.1 zeigt die Verteilung aller 213 Länder und Territorien bezüglich Einkommensgruppen und Entwicklung, untergliedert nach den Regionen der Weltgesundheitsorganisation (World Health Organization, WHO). Eine Karte, die die sechs Regionen der WHO mit ihren regionalen Büros zeigt, finden Sie auf unserer Lehrbuch-Homepage (Web-Abb. 9.1.1).

Die Mehrzahl der Länder in den Regionen Africa und South-East Asia gehört zu den am wenigsten entwickelten Ländern unserer Erde. Dagegen weisen die Industrieländer Nordamerikas und Europas sowie Australien, Neuseeland und Japan (Region Western Pacific) ein hohes Bruttonationaleinkommen und einen hohen Entwicklungsgrad auf. Die vornehmlich Öl produzierenden Länder, insbesondere des Nahen Ostens und Arabiens (Eastern Mediterranean), bilden eine Gruppe mit hohem Bruttonationaleinkommen, während China, Indien, Thailand, Brasilien und Südafrika als Beispiele für so genannte Schwellenländer mit mittlerem Einkommen stehen. Die übrigen Länder bezeichnet man als „Entwicklungsländer" oder besser "Länder mit großen Mittelknappheiten". Sie alle weisen ein niedriges Einkommen auf, viele von ihnen gehören zu den am wenigsten entwickelten Ländern der Erde. Rund 80\% der derzeit knapp sieben Milliarden Menschen leben in Entwicklungsländern. Äquatorialguinea, ein kleiner Staat in Westafrika, ist ein Sonderfall. Seit vor seinen Küsten große Erdölvorkommen entdeckt 
Tab. 9.1: Anzahl und Prozentsatz der Länder in den sechs WHO-Regionen, die sich den verschiedenen Einkommensgruppen zuordnen lassen [oben] sowie Anzahl und Prozentsatz der am wenigsten entwickelten Länder (Least Developed Counties) in diesen Regionen [unten] (Quelle: World Development Indicators. The World Bank, 2010).

\begin{tabular}{|c|c|c|c|c|c|c|}
\hline \multirow{2}{*}{$\begin{array}{l}\text { Ländergruppen } \\
\text { unterschieden nach: } \\
\text { Pro-Kopf- } \\
\text { Einkommen }\end{array}$} & \multicolumn{6}{|c|}{ WHO-Regionen } \\
\hline & $\begin{array}{l}\text { Africa } \\
(n=46)\end{array}$ & $\begin{array}{l}\text { Americas } \\
(n=42)\end{array}$ & $\begin{array}{l}\text { Eastern } \\
\text { Mediterra- } \\
\text { nean } \\
(n=25)\end{array}$ & $\begin{array}{l}\text { Europe } \\
(n=58)\end{array}$ & $\begin{array}{l}\text { South-East } \\
\text { Asia } \\
(n=11)\end{array}$ & $\begin{array}{l}\text { Western } \\
\text { Pacific } \\
(n=31)\end{array}$ \\
\hline High-income & $\begin{array}{l}1^{\mathrm{a}} \\
(2,2 \%)\end{array}$ & $\begin{array}{l}12 \\
(28,6 \%)\end{array}$ & $\begin{array}{l}8 \\
(32,0 \%)\end{array}$ & $\begin{array}{l}36 \\
(62,1 \%)\end{array}$ & $\begin{array}{l}0 \\
(0,0 \%)\end{array}$ & $\begin{array}{l}12 \\
(38,7 \%)\end{array}$ \\
\hline $\begin{array}{l}\text { Upper } \\
\text { middle-income }\end{array}$ & $\begin{array}{l}7 \\
(15,2 \%)\end{array}$ & $\begin{array}{l}20 \\
(47,6 \%)\end{array}$ & $\begin{array}{l}4 \\
(16,0 \%)\end{array}$ & $\begin{array}{l}13 \\
(22,4 \%)\end{array}$ & $\begin{array}{l}0 \\
(0,0 \%)\end{array}$ & $\begin{array}{l}4 \\
(12,9 \%)\end{array}$ \\
\hline $\begin{array}{l}\text { Lower } \\
\text { middle-income }\end{array}$ & $\begin{array}{l}10 \\
(21,7 \%)\end{array}$ & $\begin{array}{l}9 \\
(21,4 \%)\end{array}$ & $\begin{array}{l}11 \\
(44,0 \%)\end{array}$ & $\begin{array}{l}7 \\
(12,1 \%)\end{array}$ & $\begin{array}{l}7 \\
(63,6 \%)\end{array}$ & $\begin{array}{l}12 \\
(38,7 \%)\end{array}$ \\
\hline Low-income & $\begin{array}{l}28 \\
(60,9 \%)\end{array}$ & $\begin{array}{l}1 \\
(2,4 \%)\end{array}$ & $\begin{array}{l}2 \\
(8,0 \%)\end{array}$ & $\begin{array}{l}4 \\
(36,4 \%)\end{array}$ & $\begin{array}{l}4 \\
(36,4 \%)\end{array}$ & $\begin{array}{l}3 \\
(9,7 \%)\end{array}$ \\
\hline
\end{tabular}

\section{Entwicklungsstand}

$\begin{array}{lllllll}\text { Least developed } & 30^{\mathrm{b}} & 1^{\mathrm{c}} & 5^{\mathrm{d}} & 0 & 6^{\mathrm{e}} & 7^{\mathrm{f}} \\ & (65,2 \%) & (2,4 \%) & (20,0 \%) & (0,0 \%) & (54,6 \%) & (22,6 \%)\end{array}$

a Äquatorialguinea

b Angola, Benin, Burkina Faso, Burundi, Zentralafrikanische Republik, Tschad, Komoren, Kongo (Dem. Rep.), Äquatorialguinea, Eritrea, Äthiopien, Gambia, Guinea, Guinea-Bissau, Lesotho, Liberia, Madagaskar, Malawi, Mali, Mauritanien, Mosambik, Niger, Ruanda, Senegal, Sierra Leone,

São Tomé und Principe, Tansania, Togo, Uganda, Sambia.

c Haiti

d Afghanistan, Dschibuti, Somalia, Sudan, Yemen

e Bangladesh, Bhutan, Malediven, Myanmar, Nepal, Osttimor

f Kambodscha, Kiribati, Laos, Samoa, Salomon Inseln, Vanuatu, Tuvalu

und von internationalen Ölfirmen genutzt werden, ist hier das Bruttonationaleinkommen pro Kopf rasant gestiegen. Das Land gehört heute zu den High-income-Ländern, ist jedoch aufgrund der hohen ökonomischen Verwundbarkeit durch die Abhängigkeit vom Öl sowie der geringen Investitionen in Bildung und Gesundheit eines der am wenigsten entwickelten Länder der Erde. 


\subsubsection{Gesundheitsindikatoren}

Gesundheitsindikatoren sind Parameter, die Rückschlüsse auf die Gesundheit der Bevölkerung, die Gesundheitsversorgung und auf verfügbare Ressourcen erlauben.

Hier einige Beispiele:

- Ein wichtiger Indikator ist die Mortalität (s. Kap. 2.2.3). Jedes Jahr sterben weltweit ca. 57 Mio. Menschen. Davon sind 45 Mio. Erwachsene (> 15 Jahre) und 1,5 Mio. Kinder und Jugendliche im Alter von 5-15 Jahren. 9 Mio. Todesfälle entfallen auf die unter 5-Jährigen.

- Auf der Basis der altersspezifischen Mortalitätsraten lässt sich die Lebenserwartung (s. Kap. 2.2.4) eines Menschen berechnen. Dieser Wert gibt die durchschnittliche Anzahl an Jahren an, die ein Mensch eines bestimmten Alters aufgrund der aktuellen Sterberaten erwartungsgemäß noch leben würde.

- Die Lebenserwartung bei Geburt wird besonders in ärmeren Ländern maßgeblich von der Säuglingssterblichkeit (s. Kap. 2.2.3) beeinflusst.

- Indikatoren für die Morbidität in einer Bevölkerung können die Tuberkulose- oder die Malaria-Inzidenz sein. Da aber auch in vielen ärmeren Ländern - ebenso wie in den Industrienationen - die Zahl an chronischen, nicht übertragbaren Erkrankungen zunimmt, ist z.B. auch die alters- und geschlechtsspezifische Prävalenz des Diabetes mellitus Typ 2 ein wichtiger Morbiditätsindikator (Inzidenz und Prävalenz s. Kap. 2.1.2).

- Wichtige Indikatoren der Gesundheitsversorgung sind z. B. der Anteil an Einjährigen, der gegen Masern geimpft wurde oder der Prozentsatz der Geburten, die durch ausgebildetes Personal betreut wurden. Bei den älteren Menschen ist ein solcher Indikator beispielsweise der Anteil der Menschen mit Bluthochdruck, deren Blutdruck korrekt eingestellt ist.

- Zugang zu sauberem Wasser (s. Kap. 5.1) und zu Bildung sind weitere wesentliche Indikatoren für die Entwicklung eines Landes.

Darüber hinaus versucht man mit dem Konzept des Burden of Disease die Krankheitslast zu erfassen, der eine Population ausgesetzt ist. Zu dieser Krankheitslast gehören Einschränkungen durch Krankheit, Unfälle und Behinderungen ebenso wie der frühzeitige Tod. Der Gesundheitszustand einer Population wird dabei mit der Idealsituation verglichen, in der alle Mitglieder bei guter Gesundheit altern würden. Die Krankheitslast wird in Disability Adjusted Life Years (DALYs) angegeben, wobei ein DALY einem durch Erkrankung oder vorzeitigen Tod verlorenen gesunden Lebensjahr entspricht (s.a. Kap. 2.2.5).

Ein ähnlicher Gedanke liegt der Healthy Life Expectancy (HALE) zugrunde. Die HALE entspricht der Anzahl an Jahren, die bei guter Gesundheit verbracht werden können.

Auch DALYs und HALE können wichtige Gesundheitsindikatoren sein. Tab. 9.2 zeigt dies für ausgewählte Länder innerhalb der von der Weltbank definierten Einkommensgruppen. 
Tab. 9.2: Verschiedene Gesundheitsindikatoren (Mortalität, DALYs, Lebenserwartung, HALE) in ausgewählten Ländern (Quelle: Global Health Observatory Database. WHO, 2010).

\begin{tabular}{|c|c|c|c|c|}
\hline \multirow[t]{2}{*}{$\begin{array}{l}\text { Einkommensgruppe } \\
\text { Ausgewählte Länder }\end{array}$} & $\begin{array}{l}\text { Mortalitätsraten } \\
\text { (pro } 1.000 \text { Lebendgeburten bzw. } \\
\text { pro } 100.000 \text { Einwohner) }\end{array}$ & $\begin{array}{l}\text { Alters- } \\
\text { standardi- } \\
\text { sierte* } \\
\text { DALYs } \\
\text { (pro } \\
100.000 \\
\text { Einwohner }\end{array}$ & $\begin{array}{l}\text { Lebenser- } \\
\text { wartung } \\
\text { bei der } \\
\text { Geburt } \\
\text { (Jahre) }\end{array}$ & $\begin{array}{l}\text { Lebenser- } \\
\text { wartung in } \\
\text { Gesundheit } \\
=\text { HALE } \\
\text { (Jahre) }\end{array}$ \\
\hline & Säuglinge Kinder & & & \\
\hline
\end{tabular}

\section{High-income (OECD)}

- Deutschland

- Österreich

- Schweiz

- USA

$\begin{array}{rrrrrr}4 & 4 & 78 & 10.081 & 80 & 73 \\ 4 & 4 & 75 & 10.223 & 80 & 72 \\ 4 & 5 & 60 & 9.277 & 82 & 75 \\ 7 & 8 & 107 & 12.844 & 78 & 70\end{array}$

High-income (non OECD)

- Kroatien

- Saudi-Arabien

- Singapur

Higher middle-income

- Brasilien

- Südafrika

- Türkei

Lower middle-income

- Bolivien

- Philippinen

- Indien

Low-income

- Bangladesch

- Haiti

- Malawi

18

4

$18 \quad 2$

2

$\begin{array}{rr}5 & 115 \\ 21 & 154 \\ 3 & 64\end{array}$

$12.531 \quad 76$

68

$17.639 \quad 72$

62

$10.111 \quad 82$

72

$\begin{array}{ll}18 & 22 \\ & 158\end{array}$

$20.112 \quad 73$

64

$\begin{array}{lll}48 & 67 & 520\end{array}$

20

$22 \quad 150$

$46.237 \quad 53$

48

$16.307 \quad 74$

66

$\begin{array}{llllll}46 & 54 & 196 & 25.423 & 67 & 58 \\ 26 & 32 & 174 & 21.603 & 70 & 62 \\ 52 & 69 & 213 & 27.825 & 64 & 56\end{array}$

Daten von 2008, 2007 (HALE) bzw. 2004 (DALY)

* Standardisiert auf Global Standard Population der WHO.

DALY: Disability Adjusted Life Years; HALE: Healthy Life Expectancy

\subsubsection{Kinder- und Säuglingssterblichkeit}

Mehr als $90 \%$ aller Todesfälle im Kindesalter ereignen sich in der Gruppe der unter 5-Jährigen (Kindersterblichkeit), und 99\% dieser Todesfälle ereignen sich in Entwicklungsländern. Abb. 9.1 zeigt den Verlauf der Kindersterblichkeit in den Jahren 19702009 in Abhängigkeit vom Einkommen des jeweiligen Landes. 
Seit 1970 ist die Kindersterblichkeit weltweit um ca. 60\% gesunken, wobei eine Abnahme in allen Einkommensgruppen zu verzeichnen war. Die Unterschiede zwischen den Einkommensgruppen sind in absoluten Zahlen kleiner geworden, relativ gesehen haben sie jedoch deutlich zugenommen. In afrikanischen Ländern hat sich die Abnahme der Sterblichkeit durch die HIV/AIDS-Epidemie (s. a. Kap. 8.3.2) verlangsamt. In Ruanda kam es im Zusammenhang mit dem Genozid im Jahre 1994 zu einem massiven Anstieg der Kindersterblichkeit.

Bei den unter 5-Jährigen sind nur einige wenige Ursachen für eine große Anzahl der Todesfälle verantwortlich (Tab. 9.3). In den Low-income-Ländern stehen mit Pneumonien, Durchfallerkrankungen und Malaria v.a. Infektionskrankheiten, aber auch perinatale Ursachen wie Asphyxie und Sepsis im Vordergrund, während in Industrienationen extreme Frühgeburtlichkeit und Fehlbildungen dominieren.

Gegen Infektionskrankheiten wie Masern und Tetanus sind heute Impfstoffe vorhanden, sodass diese Erkrankungen in den Industrienationen nur noch relativ selten auftreten. Hier gibt es allerdings recht große Unterschiede zwischen einzelnen Ländern. So konnte das WHO-Ziel, die Masern bis 2010 in allen europäischen Staaten auszurotten, nur in Finnland erreicht werden. In vielen Entwicklungsländern spielen Infektionskrankheiten jedoch eine wesentlich bedeutendere Rolle, insbesondere wenn die Durchimpfungsraten auf Grund eines schwach entwickelten Gesundheitssystems niedrig sind.

Malaria ist in der Gruppe der Entwicklungsländer die vierthäufigste Todesursache bei den unter 5-Jährigen und hat dort zudem einen großen Anteil (30-50\%) an der Gesamtmorbidität. In den afrikanischen Ländern, in denen Malaria endemisch vorkommt, ist die Krankheit durch die immer wiederkehrenden Infektionen und die damit verbundene Anämie für ein Drittel aller DALYs bei den unter 5-Jährigen verantwortlich (s.a. Kap. 8.3).

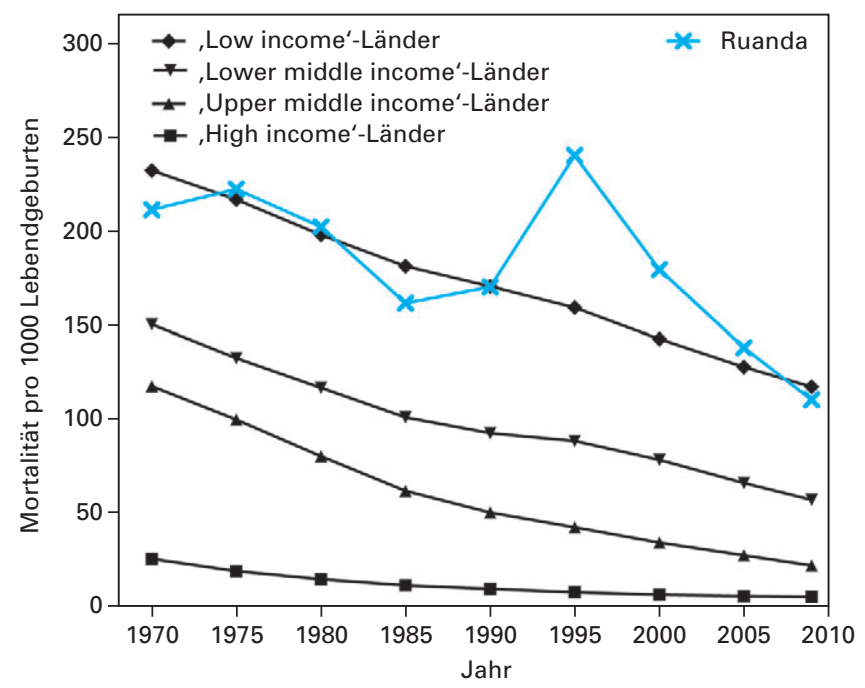

Abb. 9.1: Verlauf der Kindersterblichkeit in 194 nach ihrem Einkommen gruppierten Ländern (1970 bis 2009). Die blaue Kurve zeigt den besonderen Verlauf in Ruanda (Quelle: World Development Indicators. The World Bank, 2010). 
Tab. 9.3: Verteilung der häufigsten Todesursachen bei Kindern unter 5 Jahren im Jahr 2008 global gesehen und unterschieden nach einkommensabhängigen Ländergruppen (Quelle: World Health Organization. World Health Statistics 2010).

Todesursache

Prozent aller Todesfälle (Rang)

\begin{tabular}{lllll}
\hline Low income & Lower & Upper & High & Global \\
middle & middle & income & \\
income & income & & \\
& & &
\end{tabular}

\begin{tabular}{lccrrr}
\hline Pneumonie & $18 \%(1)$ & $19 \%(1)$ & $12 \%(3)$ & $4 \%(5)$ & $18 \%(1)$ \\
Durchfallerkrankung & $18 \%(1)$ & $14 \%(2)$ & $6 \%(6)$ & $1 \%(7)$ & $15 \%(2)$ \\
Frühgeburtlichkeit & $10 \%(4)$ & $13 \%(3)$ & $20 \%(1)$ & $27 \%(1)$ & $12 \%(3)$ \\
Geburtskomplikationen & $8 \%(5)$ & $10 \%(4)$ & $9 \%(5)$ & $6 \%(4)$ & $9 \%(4)$ \\
Malaria & $12 \%(3)$ & $7 \%(5)$ & $0 \%(9)$ & $0 \%(8)$ & $8 \%(5)$ \\
Neonatale Sepsis & $6 \%(6)$ & $6 \%(6)$ & $4 \%(8)$ & $2 \%(6)$ & $6 \%(6)$ \\
Fehlbildungen & $3 \%(7)$ & $4 \%(7)$ & $14 \%(2)$ & $25 \%(2)$ & $4 \%(7)$ \\
Unfälle & $3 \%(7)$ & $3 \%(8)$ & $5 \%(7)$ & $9 \%(3)$ & $3 \%(8)$ \\
HIV/AIDS & $3 \%(7)$ & $1 \%(10)$ & $10 \%(4)$ & $0 \%(8)$ & $2 \%(9)$ \\
Masern & $1 \%(10)$ & $2 \%(9)$ & $0 \%(9)$ & $0 \%(8)$ & $1 \%(10)$ \\
Andere Ursachen & $18 \%$ & $21 \%$ & $20 \%$ & $25 \%$ & $20 \%$ \\
\hline
\end{tabular}

Die Säuglingssterblichkeit umfasst alle Todesfälle im Zeitraum von der Geburt bis zum ersten Geburtstag (s. Kap. 2.2.3). Die Säuglingssterblichkeitsraten in Deutschland, Österreich und der Schweiz gehören zu den niedrigsten der Welt. In Deutschland sank die Rate von über 30 Todesfällen pro 1.000 Neugeborene Anfang der 1960er Jahre um fast $90 \%$ auf 4 Todesfälle pro 1.000 Neugeborene im Jahre 2008. Im selben Zeitraum ging die Säuglingssterblichkeit in Brasilien um 84 \% zurück, während in Haiti nur ein Rückgang um $65 \%$ verzeichnet wurde. In der Zwischenzeit dürfte diese Zahl in Haiti in der Folge des Erdbebens von 2010 jedoch wieder angestiegen sein. Die höchste Säuglingssterblichkeit weltweit haben derzeit (2011) Angola, Sierra Leone und Afghanistan zu verzeichnen. In Deutschland und anderen Industrieländern lässt sich die Säuglingssterblichkeit kaum noch senken, da hier ein Drittel aller Todesfälle bei Säuglingen v. a. auf angeborene Fehlbildungen zurückzuführen ist. Anders dagegen in Lowincome-Ländern, wo ein Großteil der frühen Todesfälle durch Verbesserungen der Infrastruktur und des Gesundheitswesens zu verhindern wären.

Die unterschiedlichen Säuglingssterblichkeitsraten korrelieren stark mit dem Einkommen und Entwicklungsstand der jeweiligen Länder. Aber es gibt auch Ausnahmen. So ist die Säuglingssterblichkeit in Kuba beispielsweise gleich hoch wie in den USA ( 7 pro 1.000 Neugeborene). Allerdings variiert die Rate innerhalb der USA zwischen 3,8 und 10,6 pro 1.000 Neugeborene. Diese Unterschiede sind Folge großer ethnischer und sozio-ökonomischer Gegensätze, sowie der damit verbundenen Ungleichheit im Zugang zur medizinischen Versorgung. In den USA ist also die Verteilungsgerechtigkeit (Equity, s. Kap. 1.3.2 und 3.1.2) nicht gewährleistet. In der kubanischen Gesellschaft sind diese Unterschiede weit geringer. Gleichzeitig werden in den USA öfter als in Kuba medizinische Maßnahmen bei untergewichtigen Neugeborenen ergriffen. Sie erhöhen 
zwar die Überlebensrate unmittelbar nach der Geburt, führen aber zu einem Ansteigen der Säuglingssterblichkeitsrate im weiteren Verlauf, da stark Untergewichtige in den ersten Lebenswochen ein höheres Sterberisiko aufweisen.

\subsubsection{Mortalität und Morbidität im Erwachsenenalter}

Auch bei den Erwachsenen ist die Mortalität in den letzten Jahrzehnten weltweit gesunken, wobei wie bei den Kindern große Unterschiede zwischen den einzelnen Ländern bestehen (Abb. 9.2). Russland bildet hier eine Ausnahme. Dort hat die Sterblichkeit seit 1990 sowohl bei Männern und als auch bei Frauen zugenommen (s. Box 9.1.2 mit einer Diskussion der Ursachen).
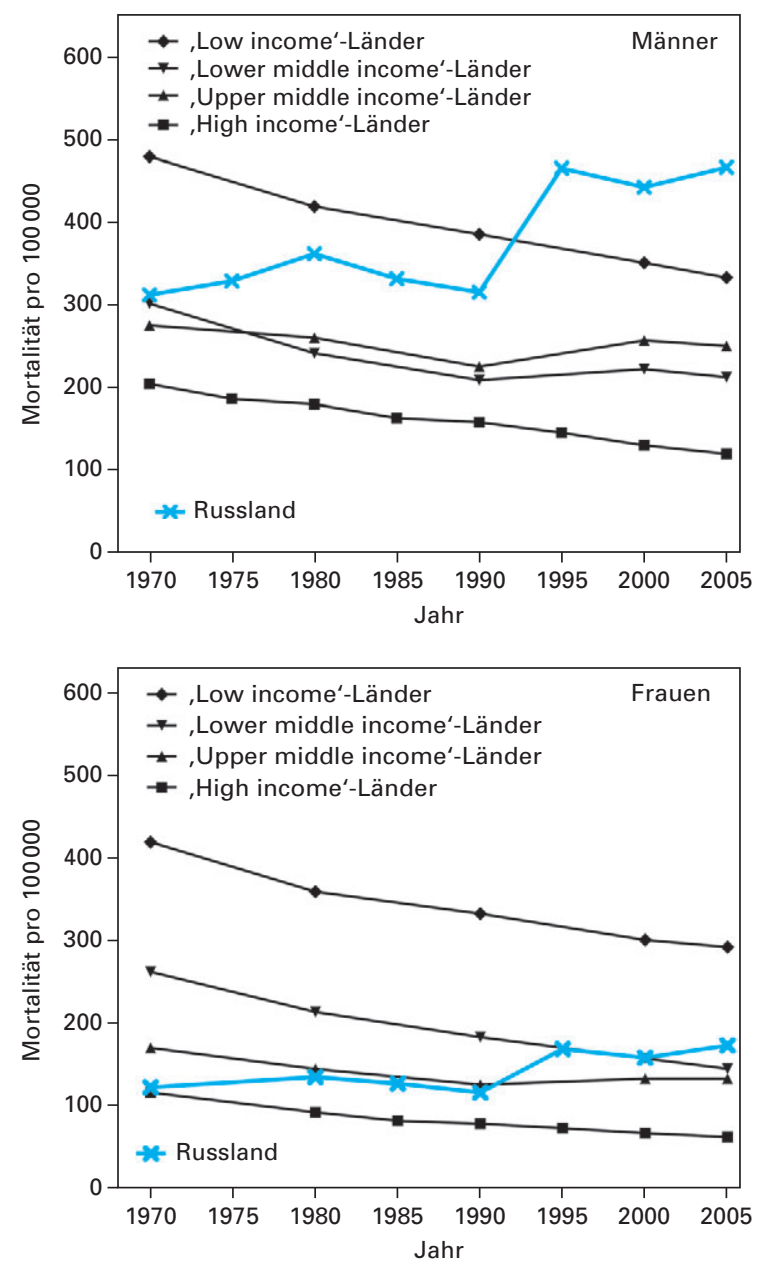

Abb. 9.2: Verlauf der altersstandardisierten Mortalität bei Erwachsenen im Alter von 15-60 Jahren in den nach ihrem Einkommen gruppierten Ländern (1970 bis 2009). Die blaue Kurve zeigt den besonderen Verlauf in Russland (Russische Sozialistische Föderative Sowjetrepublik, seit 1992 russischen Föderation) (Quelle: World Development Indicators. The World Bank, 2010). 
Box 9.1.2: Eau de Cologne, „Zapoi“ und die Mortalität russischer Männer.

2008 lag die Lebenserwartung für Männer in Russland mit 62 Jahren deutlich unter dem Durchschnitt aller Upper-middle-income-Länder (68 J.) und war damit niedriger als die Lebenserwartung in einigen Low-income-Ländern, wie z. B. in Bangladesh (65 J.) oder in Nepal (66 J.). Wichtigste Ursache hierfür ist wahrscheinlich die hohe Mortalität durch Alkoholvergiftungen. In einer Industriestadt im Ural wurden in Rahmen einer Fall-Kontroll-Studie die Trinkgewohnheiten von verstorbenen Männern erfasst ${ }^{a}$. Die Angaben wurden dann mit denjenigen von lebenden Männern gleichen Alters verglichen, die den Verstorbenen in Bildungsstand und Rauchgewohnheiten entsprachen.

Das Nationalgetränk Wodka war etwa gleich häufig von Verstorbenen und Kontrollpersonen konsumiert worden. Beide Gruppen unterschieden sich jedoch in ihren Trinkgewohnheiten. 45 Prozent der Verstorbenen, aber nur 12 Prozent der Kontrollpersonen waren als problematische Trinker eingestuft worden. Zu den problematischen Trinkgewohnheiten gehört in Russland das „Zapoi“. Hierunter versteht man mehrtägige Alkoholexzesse, in denen die Männer sich dem normalen sozialen Leben entziehen. Problematisches Trinken verdreifachte das Sterberisiko. Noch stärker war der Zusammenhang mit dem Konsum von nicht für den Verzehr vorgesehenen Alkoholika, z. B. Eau de Cologne. Wodka enthält 43 Prozent Alkohol. In Eau de Cologne, Parfüm, medizinischen Tinkturen und Reinigungsmitteln sind es bis zu 97 Prozent. Der Verkauf dieses Alkohols ist steuerfrei, sodass die genannten Substanzen bis zu sechsmal weniger kosten als Wodka.

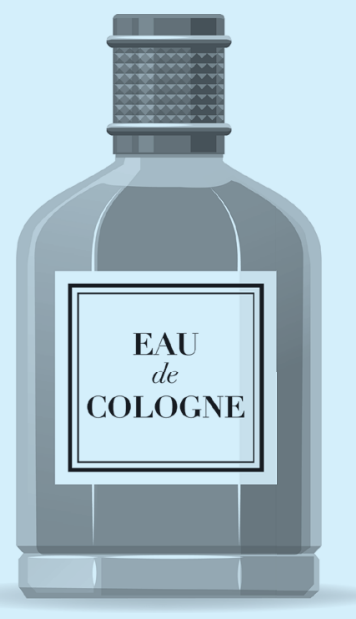

Männer der untersten Bildungsschicht konsumieren solche Substanzen im Vergleich zu Männern aus höheren Schichten etwa 8-mal so oft und haben etwa 5-mal so oft einen Zapoib. Bei 41 Prozent der Verstorbenen und 8 Prozent der Kontrollpersonen war das Trinken von derartigem „Billig-Alkohol“ angegeben worden. Unter Berücksichtigung des Alters der Personen errechneten Epidemiologen hieraus für sie ein mehr als 9-Mal so hohes Sterberisiko. Aufgrund der zitierten Studie schätzt man, dass etwa $40 \%$ aller Todesfälle bei Männern in Russland auf Alkoholkonsum zurückzuführen sind.

a Leon DA et al. Alcohol consumption and public health in Russia. Lancet 2007; 369: 2001-2009

b Tomkins S et al. Prevalence and socio-economic distribution of hazardous patterns of alcohol drinking: study of alcohol consumption in men aged 25-54 years in Izhevsk, Russia. Addiction 2007; 102: 544-553

Im Gegensatz zu den Todesursachen bei den Kindern ist die Mehrzahl der Todesfälle bei Erwachsenen auf nicht übertragbare Krankheiten zurückzuführen. Dazu gehören vor allem Herz-Kreislauf-Erkrankungen (s. Kap. 7.1), bösartige Tumore (s. Kap. 7.2), chronische Atemwegserkrankungen (s. Kap. 7.6) und der Diabetes mellitus (Zuckerkrankheit; s. Kap. 7.5). 
Mit sinkender Geburtenrate und steigender Lebenserwartung erhöht sich in vielen Ländern der Anteil der erwachsenen, insbesondere der älteren Bevölkerung. In solchen Populationen dominiert zunehmend die Krankheitslast durch chronische Krankheiten. Man bezeichnet diesen Vorgang als epidemiologischen Übergang oder epidemiologische Transition. Deutschland, die Schweiz und andere Industrienationen haben im letzten Jahrhundert schon weite Strecken dieses Übergangs durchlebt. Dort sind heute $90 \%$ der Todesfälle auf nicht übertragbare Krankheiten zurückzuführen. In Malawi und vielen anderen Least Developed Countries hat die epidemiologische Transition erst begonnen, während sie in Brasilien und anderen Schwellenländern schon weiter vorangeschritten ist. Die epidemiologische Transition bedeutet für Schwellenländer eine doppelte Krankheitslast mit hohen Raten bei den Infektionskrankheiten und zunehmender Belastung durch chronische Krankheiten. Während Infektionskrankheiten weiterhin vor allem in ländlichen Gegenden und den Elendsvierteln der Städte vorkommen, stellen chronische Krankheiten sowohl ein Problem der ländlichen Bevölkerung (v. a. durch Bluthochdruck, Schlaganfall, s. Kap. 7.1) als auch zunehmend der Mittel- und Oberschicht in den Städten (v. a. durch Bösartige Tumore, Lungen- und Herz-Kreislauf-Erkrankungen; s. Kap. 7.1, 7.2 und 7.6) dar.

Abb. 9.3 zeigt die Krankheitslast in DALYs für Männer und Frauen global gesehen und unterschieden nach Ländern mit verschiedenem Einkommen. Sichtbar ist dabei auch jeweils der Anteil, den Infektionskrankheiten, nicht übertragbare Krankheiten und Unfälle/Verletzungen hierbei einnehmen. Im Jahr 2004 wurde die weltweite Krankheitslast für Erwachsene auf 975 Millionen DALYs, d. h. verlorene gesunde Lebensjahre geschätzt (für alle Altersgruppen waren es 1,52 Milliarden DALYs). Von dieser Krankheitslast entfielen 418 Millionen auf Low income-Länder (42,9\%), 445 Millionen auf Middle income-Länder (45,6\%) und 112 Millionen (11,5\%) auf High income-Länder. Die im Erwachsenenalter dominierenden nicht übertragbaren Krankheiten sind auch in Low-income-Ländern für die Mehrheit der DALYs bei Erwachsenen verantwortlich. Allerdings nimmt ihre Bedeutung mit dem jeweiligen Einkommen des Landes zu, wäh-

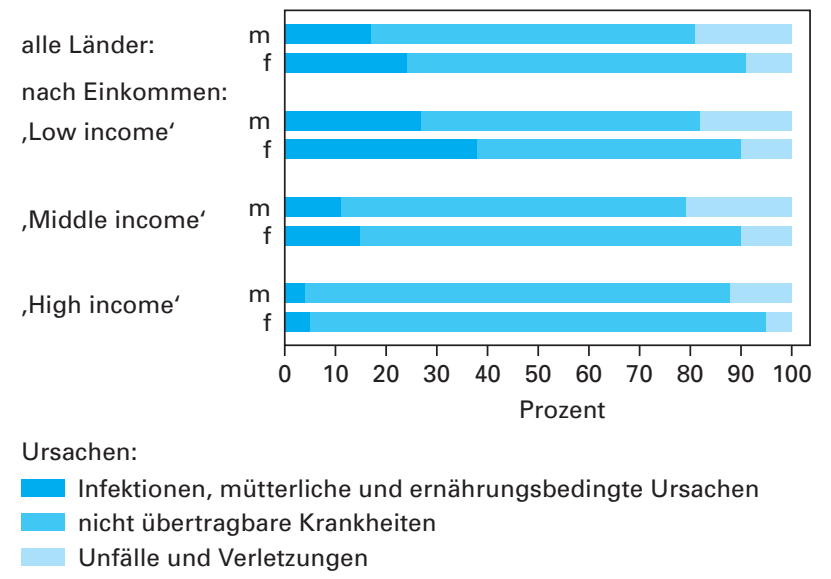

Abb. 9.3: Prozentuale Verteilung der Krankheitslast in DALYs (verlorene gesunde Lebensjahre) global und nach einkommensabhängigen Ländergruppen. Die Daten aus dem Jahr 2004 unterscheiden jeweils zwischen Männern $(\mathrm{m})$ und Frauen $(\mathrm{f})$ und beziehen sich auf die Altersgruppe der über 15-Jährigen (Quelle: Global Health Observatory Database. WHO, 2010). 
rend Infektionen, mütterliche und ernährungsbedingte Ursachen mit zunehmendem Einkommen anteilsmäßig weniger wichtig werden. Berücksichtigt man alle Altersgruppen, sind die letztgenannten Ursachen in den Low-income-Ländern jedoch mit $57 \%$ aller DALYs noch immer die wichtigsten. In den Middle-income- und Low-income-Ländern fallen darüber hinaus Unfälle und Verletzungen besonders ins Gewicht, Männer sind hier wesentlich häufiger betroffen als Frauen (s. a. Kap. 7.8).

\subsubsection{Die weltweit wichtigsten Ursachen der Krankheitslast}

Je nach dem Einkommen eines Landes zeigen sich große Unterschiede in der Verteilung der wichtigsten Erkrankungen, die dann wiederum Auswirkungen auf die DALYs einer Bevölkerung haben. Web-Tab. 9.1.1 auf unserer Lehrbuch-Homepage zeigt die jeweils zehn wichtigsten Ursachen für DALYs in allen Altergruppen weltweit sowie gegliedert nach dem Einkommen der Länder. Es fällt auf, dass die wichtigsten Ursachen in den Low-income-Ländern ähnlich denen sind, wie sie in der Welt insgesamt auftreten. Von großer Bedeutung sind hier u.a. Lungenentzündungen, Durchfallerkrankungen, HIV/ AIDS, Frühgeburtlichkeit und ischämische Herzkrankheiten. Ausnahmen sind Malaria und Tuberkulose, die weltweit nicht mehr zu den zehn wichtigsten Ursachen gehören. In den High-income-Ländern dominieren dagegen die nicht übertragbaren Krankheiten. Hier gehören unipolare Depression und Hörverlust zu den wichtigsten Ursachen für verlorene gesunde Lebensjahre im Erwachsenenalter, obwohl sie nicht mit einer hohen Sterblichkeit assoziert sind. Weltweit sind etwa 40\% der DALYs auf solche Einschränkungen durch nicht-tödliche Erkrankungen oder Verletzungen zurückzuführen. 60\% betreffen vorzeitige Todesfälle.

In den High-income-Ländern steht mit der unipolaren Depression eine psychiatrische Erkrankung an erster Stelle. Die lange unterschätzte Dimension dieser Erkrankungsgruppe sowie Näheres zu Verteilung, Risiken und Ursachen psychiatrischer Krankheiten in den verschiedenen Gesellschaften ist im World Health Report - Mental Health der WHO dargestellt. Der hohe Anteil an psychischen Ursachen unter den DALYs in Highincome-Ländern unterstreicht den Einfluss sozialer (Drogenmissbrauch, Gewalt, Missbrauch, Diskriminierung) und ökonomischer Faktoren (Arbeitslosigkeit, Armut, ungenügende Bildung, Stress am Arbeitsplatz), die hier wirken. Alkoholassoziierte Erkrankungen spielen ebenfalls eine große Rolle (s.a. Kap. 7.7). Weitere wichtige Ursachen in den High income-Ländern sind kardiovaskuläre Erkrankungen und verschiedene Formen der Demenz.

Die Liste der zehn wichtigsten Ursachen in den Middle-income-Ländern stellt eine Kombination aus denjenigen der Low income- und der High income-Länder dar. Hier führen die nicht übertragbaren Erkrankungen die Liste an, aber HIV/AIDS, Pneumonien und Durchfallerkrankungen finden sich weiterhin unter den Top Ten. Auch nicht-korrigierte Ametropien (Kurz- und Weitsichtigkeit) sind in den Middle-income-Ländern eine wichtige Ursache für verlorene gesunde Lebensjahre. In den Schwellenländern sorgen Verkehrsunfälle für immer mehr DALYs. Hauptgründe hierfür sind ungenügend gesicherte Straßen, fehlende Geschwindigkeitsbegrenzungen, der schlechte technische Zustand vieler Kraftfahrzeuge sowie die fehlende Gurtpflicht.

Doch auch innerhalb einer Einkommensgruppe bestehen in den dort eingruppierten Ländern zum Teil große Unterschiede. Die wichtigsten Ursachen für verlorene gesunde Lebensjahre entsprechen in Deutschland, der Schweiz und Österreich im Wesentlichen 
dem Muster der High-income-Länder. Eine Ausnahme ist der Suizid, der in der Schweiz unter den ersten zehn Ursachen (Rang 8) zu finden ist (s. Kap. 7.7). In den USA rangieren Verkehrsunfälle an fünfter, in Saudi Arabien sogar an dritter Stelle. Zwischen den Middle-income- und Low-income-Länder sind die Unterschiede in den Ursachen noch ausgeprägter. HIV/AIDS findet man in Brasilien nicht unter den zehn wichtigsten Ursachen. In Bangladesh ist die Krankheit kaum vertreten, in Haiti und den Philippinen gehört sie jedoch zu den zehn wichtigen DALYs-Ursachen. In Südafrika und Malawi (sowie anderen Länder in Subsahara-Afrika) ist sie sogar der häufigste Grund. In Brasilien und Südafrika gehören Gewalttaten zu den wichtigsten Ursachen für verlorene gesunde Lebensjahre (Rang 2 in Brasilien, Rang 3 in Südafrika).

\subsection{Determinanten der globalen Gesundheit}

Armut, Hunger, niedriger Bildungsstand, Bevölkerungswachstum und Ungleichheiten zwischen den Geschlechtern sind wichtige Determinanten der Unterschiede in der Gesundheit zwischen verschiedenen Bevölkerungsgruppen und zwischen den Bevölkerungen verschiedener Länder. Die genannten Determinanten beeinflussen den Altersaufbau einer Bevölkerung. Die daraus resultierenden Unterschiede im Altersaufbau können wiederum einen Teil der Unterschiede in der Gesundheit zwischen den Bevölkerungen der verschiedenen Länder erklären.

\subsubsection{Armut}

Etwa die Hälfte der Weltbevölkerung (ca. 3,5 Mrd. Menschen) lebt von weniger als 2 US-Dollar am Tag. Armut und schlechter Gesundheitszustand sind hier eng miteinander verwoben. Sowohl auf nationaler als auch auf individueller Ebene verhindert ein niedriges Einkommen die Verfügbarkeit, den Zugang sowie die Nutzung von und zu Gesundheitseinrichtungen. Umgekehrt trägt ein schlechter Gesundheitszustand zur Armut bei, weil kranke oder behinderte Menschen meist keiner geregelten Tätigkeit nachgehen können. Ein guter Gesundheitszustand ist somit nicht nur Voraussetzung für individuellen Wohlstand, sondern auch für wirtschaftliches Wachstum und für die Entwicklung eines Landes.

Die Web-Abb. 9.2.1 auf unserer Lehrbuch-Homepage zeigt, dass das Volkseinkommen stark mit Kindersterblichkeit und Lebenserwartung korreliert. Die höchste Lebenserwartung haben die Menschen in Ländern mit hohem Volkseinkommen (z. B. Schweiz, Deutschland, Japan, Frankreich, USA), die niedrigste Lebenserwartung findet man in Ländern mit niedrigem Volkseinkommen (z. B. in Simbabwe, Somalia, der Demokratischen Republik Kongo). Allerdings gibt es hier Ausreißer: Die Menschen in Südafrika haben z. B. eine relativ geringe Lebenserwartung im Vergleich zum dort vorhandenen Wohlstand. Dies ist auf die ungleiche Verteilung des Einkommens zurückzuführen. Eine Minderheit der Gesamtpopulation verfügt über den größten Teil des Einkommens, während die große Mehrheit der Bevölkerung in Armut lebt und eine hohe Mortalitätsrate aufweist.

Die Zusammenhänge zwischen Volkseinkommen und Kindersterblichkeit wurden nach der Deklaration von Alma Ata (1978) erstmals systematisch untersucht. Länder wie Costa Rica, Kuba und der Bundesstaat Kerala in Indien gehörten hiernach zu den „Sonderfällen“, da sie trotz geringem Einkommen eine niedrige Kindersterblichkeitsrate auf- 
wiesen. Man konnte fünf Schlüsselfaktoren identifizieren, die es einem Land erlauben, trotz relativ geringem Volkseinkommen gute Gesundheitsergebnisse zu erzielen:

- Staatliche Förderung der sozialen Sicherheit

- Einbeziehung der Bevölkerung in die Prioritätensetzung und Entscheidungsfindung (Community Participation)

- Verteilungsgerechtigkeit (Equity) durch Berücksichtigung der Risikogruppen (ethnische Minderheiten, Kinder, Frauen, Randgruppen)

- Intersektorielle Zusammenarbeit (Gesundheit, Bildung, Wasserversorgung, Landwirtschaft)

- Einbeziehung der lokalen Traditionen und Kultur

\subsubsection{Hunger}

Auf der Erde gibt es genug Nahrung, um die gesamte Weltbevölkerung zu ernähren. Dennoch leiden etwa 800 Mio. Menschen an chronischem Hunger und beinahe 200 Mio. Kinder sind untergewichtig. Die Hauptgründe für Hungersnöte sind Umsiedlungen und Zerstörungen von landwirtschaftlichen Erzeugnissen durch kriegerische Handlungen, aber zunehmend auch die Fehlverteilung von und die Spekulation mit Grundnahrungsmitteln.

Von Mangelernährung spricht man dann, wenn ein Mensch über einen längeren Zeitraum nicht genug oder zu einseitige Nahrung aufnimmt. Unterernährung ist eine Form der Mangelernährung, die durch eine anhaltende Reduktion der Energiezufuhr hervorgerufen wird. Sie führt zu einer allgemeinen Schwäche, verzögertem Wachstum und Untergewicht. Bei Kindern kommt es als Folge einer zu geringen Eiweiß-Zufuhr, kombiniert mit einem niedrigen Energiegehalt der Nahrung, zum Kwashiorkor. Kwashiorkor bedeutet in einer ghanaischen Sprache: „die Krankheit, die ein Kind bekommt, wenn ein neues Kind geboren wird". Sie tritt dann auf, wenn während einer erneuten Schwangerschaft die Milchproduktion bei der Mutter aussetzt und das Kind danach mit eiweißarmer Nahrung ernährt wird. Ein typisches Symptom des Kwashiorkors ist die Ödembildung (sichtbar v.a. am so genannten Hungerbauch). Im Gegensatz hierzu ist der Marasmus Folge einer generellen Unterernährung mit einem Mangel an Eiweißen, Fetten und Kohlenhydraten. Er führt durch den Abbau der Energie- und Eiweißreserven des Körpers zur Gewichtsabnahme bei gleichzeitiger Reduktion der Muskelmasse, nicht jedoch zur Ödembildung. Diese Form des Protein-Energie-Mangelsyndroms (PEM) findet man v. a. in den Least Devoloped Countries. Unterernährung trägt zu etwa $50 \%$ aller Todesfälle bei Kindern bei. Einer der Gründe hierfür ist die eingeschränkte Widerstandskraft der betroffenen Kinder gegen Infektionskrankheiten. Schwangere Frauen, die unterernährt sind, bringen sehr oft untergewichtige Kinder zur Welt, die dann wiederum ein stark erhöhtes Risiko haben, an Atemwegsinfektionen und Durchfall zu erkranken.

\subsubsection{Bildung}

Der Gesundheitszustand einer Bevölkerung ist eng mit ihrer Bildung verbunden. Global gesehen korreliert die Anzahl an Schuljahren mit der Lebenserwartung ähnlich eng wie das Einkommen. Auch innerhalb der einzelnen Länder ist dieser Zusammenhang nach- 
weisbar. So übertrifft die Lebenserwartung von Hochschulabgängern in der Schweiz die der am wenigsten Gebildeten um zehn Jahre (s. a. Kap. 1.3.2).

Dabei hat insbesondere in ärmeren Ländern die Ausbildung von Mädchen einen größeren Einfluss auf die Entwicklung einer Gesellschaft sowie auf die Verminderung von Armut als die Ausbildung der Jungen. Ihre Ausbildung führt zu einer positiven Verstärkung folgender Faktoren: Gut ausgebildete Frauen heiraten später, benutzen Verhütungsmittel, bekommen weniger Kinder und ziehen diese gesünder auf. Sie treffen bessere Entscheidungen für sich und ihre Kinder und leisten wirtschaftlich größere Beiträge zum Haushalt. Zudem haben Mädchen, die in eine kleinere Familie hinein geboren werden, eine größere Chance, eine Schule zu besuchen und diese auch abzuschließen.

\subsubsection{Ungleichheit zwischen den Geschlechtern}

Gesellschaftlich festgelegte Rollen und Haltungen gegenüber Frauen und Männern führen zu sozialen Unterschieden, die die eine Gruppe stärken und die andere benachteiligen. Armut und unzureichende Bildung verstärken die Ungleichheit zwischen den Geschlechtern. Weltweit sind zwei Drittel der Kinder, die keine Schule besuchen oder abschließen können, Mädchen. Frauen erhalten in vielen Ländern (auch in der Schweiz und Deutschland!) weniger Lohn für dieselbe Arbeit, der Zugang zu gut bezahlter Arbeit ist ihnen oft verwehrt. In einkommensschwachen Ländern sind Schwangerenvorsorge und Geburtshilfe meist nicht für alle Frauen zugänglich. Zudem führt die Illegalität von Abtreibungen jedes Jahr zu ca. 20 Mio. (von weltweit insgesamt 46 Mio.) Abtreibungen unter gesundheitsschädigenden Bedingungen. Nicht selten sind schwerste Infektionen, Blutungen oder Tod die Folge.

\subsubsection{Kriegerische Konflikte}

Im Jahr 2010 wurden global in 29 Ländern oder Territorien bewaffnete Konflikte ausgetragen. Anders als die beiden Weltkriege sind viele der heutigen Konflikte Bürgerkriege, die oft schon seit Jahrzehnten geführt werden, so z. B. in einer Reihe von afrikanischen Ländern wie Ruanda, Angola, Uganda und Somalia. Trotz des Schutzes, den die Genfer Konvention der Zivilbevölkerung zusichert, sind vor allem Zivilisten die Leidtragenden dieser Auseinandersetzungen. 95\% aller Toten in Bürgerkriegen sind Nichtkombattanten. Ein Krieg kann sich direkt und indirekt auf die Gesundheit einer Bevölkerung auswirken, seine Folgen sind oft über Generationen wirksam. Noch Jahre und Jahrzehnte nach einem Konflikt stellen Landminen eine große Gefahr für die Zivilbevölkerung dar (z. B. in Bosnien-Herzegowina oder Kambodscha). Die International Campaign to Ban Landmines erreichte 1997 ein Verbot von Herstellung, Handel und Einsatz von Anti-Personenminen und erhielt dafür den Friedensnobelpreis. Doch auch heute noch sind Landminen pro Jahr für rund 20.000 Todesfälle und schwere Verletzungen verantwortlich. Zudem werden sie von vielen Industrieländern trotz des Verbots weiterhin produziert.

In bürgerkriegsähnlichen Konflikten werden Frauen immer häufiger Opfer von Gewalt. So wurden Frauen in Ex-Jugoslawien, Ruanda und Sierra Leone systematisch von Soldaten oder Milizionären vergewaltigt. In der Folge führen schwerste psychische Traumatisierungen, ungewollte Schwangerschaften und die Übertragung von HIV/AIDS zu gravierenden gesundheitlichen Belastungen bei den Betroffenen. 
Darüber hinaus sind bewaffnete Konflikte Ursache für eine ökonomische Stagnation, die das Armutsrisiko bei der Bevölkerung erhöht. Die Rüstungsausgaben entziehen dem Gesundheitswesen die Ressourcen, die z. B. für Impfkampagnen oder Vektor-Kontrollmaßnahmen (s. Kap. 8.3 und Kap. 8.4) benötigt würden. In Burundi trug dieser Faktor 2001 zu einer Malaria-Epidemie bei. Ärzte und Pflegefachkräfte verlassen bei kriegerischen Konflikten oftmals ihr Land. Die Zerstörung der Infrastruktur begünstigt Epidemien von ansonsten vermeidbaren Krankheiten (z. B. Cholera), chronischer Nahrungsmangel führt zu Hungersnöten.

\subsubsection{Umweltveränderungen}

Unsere Umwelt wird durch Bevölkerungswachstum, Industrialisierung und Urbanisierung stark verändert. Treibhausgase, wie sie bei der Verbrennung von fossilen Brennstoffen entstehen, Bodenerosion, Erschöpfung der Trinkwasservorräte, Abnahme der Biodiversität und Anreicherung von Chemikalien in Ökosystemen sind einige Beispiele dafür, wie die Natur in Mitleidenschaft gezogen wird. Die Bevölkerung der Industrieländer verbraucht ca. 50 Mal mehr Energie pro Person als die Einwohner von Entwicklungsländern. Der Weltklimarat (Intergovernmental Panel on Climate Change) geht von einem Anstieg der weltweiten Durchschnittstemperatur um 1 bis $3^{\circ} \mathrm{C}$ bis zum Jahr 2100 aus. Schon heute zeigen sich zunehmend Auswirkungen des Klimawandels auf die Gesundheit der Bevölkerung (s.a. Kap. 5.5).

\subsection{Health for All: Strategien, Akteure und Setzung von Prioritäten}

Im Jahr 1977 formulierte die Weltgesundheitsversammlung der Vereinten Nationen (World Health Assembly) das Ziel, dass die gesamte Weltbevölkerung bis zum Jahr 2000 ein Leben in Gesundheit führen kann (s. Health for All). Die primäre Gesundheitsversorgung (Primary Health Care, PHC) wurde später in der Alma-Ata-Deklaration (1978) als Schlüsselstrategie (s.a. Kap. 1.6) identifiziert, mit deren Hilfe dieses Ziel zu erreichen sei.

Seither wird kontrovers diskutiert, welches die geeigneten Strategien zur Erreichung von Health for All sein könnten. In der Diskussion stehen v. a. so genannte vertikale Programme, die zentral geplant werden und sich auf wenige Gesundheitsprobleme eines Landes oder einer Region konzentrieren. Weder die in Alma Ata propagierte PHC mit dem rudimentär ausgebildeten Village Health Worker, noch punktuelle vertikale Programme werden die globalen Gesundheitsprobleme nachhaltig lösen können. Der Aufbau von geeigneten Gesundheitssystemen mit einer starken PHC-Komponente, mit Institutionen und Maßnahmen der Prävention und Gesundheitsförderung stellen wichtige gesellschaftliche Aufgaben dar, die weit über den Gesundheitssektor hinausgehen und ohne eine wirksame Bekämpfung der Armut nicht zu realisieren sind. Diese Erkenntnis hat die WHO im Weltgesundheitsbericht 2008 unter dem Titel "Primary Health Care - Now More Than Ever" festgehalten.

\subsubsection{Millennium-Entwicklungsziele}

Schon die im September 2000 von den Vereinten Nationen verabschiedeten „Millennium-Entwicklungsziele" (Millennium Development Goals, MDGs; s. Box 9.3.1) tragen 
dem Rechnung. Armutsbekämpfung, Friedenserhaltung und Umweltschutz wurden als die wichtigsten Ziele der internationalen Gemeinschaft definiert. Drei der acht Ziele haben unmittelbar mit Gesundheit zu tun: Reduktion der Kindersterblichkeit, Verbesserung der Gesundheitsversorgung der Mütter sowie Bekämpfung von HIV/AIDS, Malaria und anderen Krankheiten. Auch die übrigen Ziele sind eng mit dem Gesundheitssektor verknüpft. Die MDGs verdeutlichen, dass ein verbesserter Gesundheitszustand der Bevölkerung zum Schlüsselfaktor bei der Bekämpfung der Armut in der Welt werden kann. Um das Erreichen der Ziele messbar zu machen, wurden 20 Unterpunkte und 60 Indikatoren definiert. Als Basisjahr dient dabei das Jahr 1990. 2015 wurde als Zieljahr festgelegt. Zwar sind seit der Formulierung der Millennium-Entwicklungsziele in vielen Bereichen Fortschritte zu verzeichnen, jedoch bestehen dabei erhebliche Unterschiede zwischen den Weltregionen. So ist die WHO-Region Afrika beispielsweise weit hinter der Zielvorgabe zur Kindersterblichkeit zurückgeblieben, während Lateinamerika das Ziel voraussichtlich erreichen wird. Zur Umsetzung der MDGs werden also weitere finanzielle Mittel benötigt.

Box 9.3.1: Die Millennium-Entwicklungsziele.

1. Bekämpfung von Hunger und extremer Armut

2. Primärschulbildung für alle

3. Gleichstellung der Geschlechter und Stärkung der Rolle der Frauen

4. Senkung der Kindersterblichkeit

5. Verbesserung der Gesundheitsversorgung der Mütter

6. Bekämpfung von HIV/AIDS, Malaria und anderen schweren Krankheiten

7. Ökologische Nachhaltigkeit

8. Aufbau einer globalen Partnerschaft für Entwicklung

Eine ausführliche Version der Millennium-Ziele sowie Daten zum Stand der Zielerreichung finden Sie auf unserer Lehrbuch-Homepage.

\subsubsection{Globaler Fonds, Stiftungen und Initiativen}

Obwohl vertikale Gesundheitsprogramme immer wieder in der Kritik stehen, sind sie recht erfolgreich in der Bekämpfung von Zielkrankheiten. Ein aktuelles Beispiel ist die Bekämpfung von AIDS, Tuberkulose und Malaria durch den Global Fund to Fight AIDS, Tuberculosis and Malaria (GFATM). Der GFATM wurde im Juni 2002 auf Beschluss der UN Sondergeneralversammlung zu HIV und AIDS gegründet. Aufgabe des Fonds ist die Finanzierung von Maßnahmen zur Bekämpfung der drei Infektionskrankheiten in Low- und Middle-income-Ländern. Geldgeber sind Regierungen und Stiftungen. Seit seiner Gründung hat der Fonds Projekte und Programme mit einem Volumen von etwa 22 Mrd. US-Dollar in 150 Ländern bewilligt. Mit dieser Hilfe konnten u.a. 3 Mio. HIV-infizierte Menschen und 7,7 Mio. Tuberkulosepatienten behandelt sowie die Haushalte in den betroffenen Ländern mit 160 Mio. insektizidbehandelten Mückennetzen versorgt werden (s.a. Kap. 8.2). Zu Beginn einer Zusammenarbeit mit einem Land definieren Fonds und Empfängerorganisation gemeinsam die zu erreichenden Ziele und legen Kriterien fest, wie diese zu messen sind. Programme, die vereinbarte Zielvorgaben wesentlich unterschreiten, erhalten keine weiteren Zahlungen mehr. 
Die Bill \& Melinda Gates Foundation (BMGF) ist mit einem Vergabevolumen von zwei bis drei Mrd. US-Dollar pro Jahr die größte private Stiftung der Welt. Sie unterstützt die Behandlung und Bekämpfung von Krankheiten in der ganzen Welt mit einem Fokus im Bereich AIDS, Tuberkulose und Malaria. Außerdem engagiert sie sich im Bereich der Entwicklung und Bereitstellung von Impfstoffen und Impfstofftechnologien. Die Globale Allianz für Impfstoffe und Immunisierung (Global Alliance for Vaccine and Immunization, GAVI) wird zu 75\% (das sind ca. 1,5 Mrd. US-Dollar pro Jahr) von der BMGF finanziert. GAVI ist ein Beispiel für eine der vielen globalen Gesundheitsinitiativen (Global Health Initiatives, GHI), globalen Partnerschaften und Programmen, die im Zusammenhang mit der Umsetzung der Millennium-Entwicklungsziele entstanden sind. Weitere Beispiele sind die Stop TB Partnership, Roll Back Malaria, Partnership for Maternal, Newborn and Child Health sowie die Health Workforce Alliance, die sich für eine Stärkung der Personalstruktur im Gesundheitswesen einsetzt.

\subsubsection{Die WHO und andere internationale Organisationen}

Die Weltgesundheitsorganisation (WHO) ist eine Sonderorganisation der Vereinten Nationen (UN) mit 193 Mitgliedstaaten und sechs Regionen (s. Web-Abb. 9.1.1 auf unserer Lehrbuch-Homepage). Als Koordinationsbehörde für das internationale öffentliche Gesundheitswesen unterstützt sie Entwicklungsländer beim Aufbau von Gesundheitssystemen und koordiniert nationale und internationale Aktivitäten, wie z. B. globale Impfprogramme und Programme gegen übertragbare Krankheiten, Rauchen oder Übergewicht. Ein weiterer Schwerpunkt ist die weltweite Erhebung und Analyse von Gesundheits- und Krankheitsdaten. Die wichtigste Publikation der WHO ist der jährlich erscheinende Weltgesundheitsbericht (World Health Report), der in jedem Jahr auf ein aktuelles Thema der Globalen Gesundheit eingeht und dazu die wesentlichen globalen Daten veröffentlicht. Das jährliche Budget der WHO beträgt etwa 2 Mrd. US-Dollar.

Weitere wichtige internationale Organisationen, die sich mit Gesundheit beschäftigen, sind z. B. die UNAIDS (Joint United Nations Programme on HIV/AIDS; ein Projekt der Vereinten Nationen, dessen Ziel es ist, die verschiedenen Aktivitäten der Ländern im Kampf gegen HIV/AIDS zu koordinieren), die IARC (International Agency for Research on Cancer, eine Forschungseinrichtung der WHO zum Thema Krebs) und The Union (International Union Against Tuberculosis and Lung Disease, eine Organisation der nationalen Lungenligen).

Die WHO und andere Organisationen der UN sind angesichts der vielen Akteure im Bereich Gesundheit stark gefordert. Im Zentrum steht dabei die Harmonisierung der Maßnahmen von mehr als $100 \mathrm{GHI}$, von globalen und regionalen Finanzierungsagenturen sowie Projekten der Entwicklungszusammenarbeit.

\section{Internet-Ressourcen}

Auf unserer Lehrbuch-Homepage (www.public-health-kompakt.de) finden Sie Links zu den genannten Institutionen, zu weiterführender Literatur sowie zu anderen themenrelevanten Internet-Ressourcen. Besuchen Sie z. B. die Seiten von Hans Rosling, der internationale Statistiken interaktiv zum Leben bringt, oder machen Sie sich ein Bild davon, welche Strecke wir bereits auf dem Weg zur Erreichung der Millennium-Entwicklungsziele zurückgelegt haben. 
